\title{
La ganadería familiar, elemento cotidiano de los traspatios de la comunidad Juan Nepomuceno Álvarez, Copala, Guerrero, México
}

\author{
Alejandro García Flores ${ }^{1,2, a}$ \& Elsa Guzmán Gómez ${ }^{1 *}$ \\ ${ }^{1}$ Posgrado en Ciencias Agropecuarias y Desarrollo Rural, Facultad de Ciencias Agropecuarias $\&{ }^{2}$ Centro de \\ Investigaciones Biológicas. Universidad Autónoma del Estado de Morelos, México.
}

\begin{abstract}
Resumen - Este trabajo tiene como objetivo conocer la importancia cultural y económica de la ganadería familiar como sistema de producción campesina en las familias de la comunidad Juan Nepomuceno Álvarez. Se aplicaron 53 encuestas en unidades familiares. El sistema de producción es tradicional, con trabajo familiar, para fines de autoconsumo de la producción de carne y huevo (91\%), y venta (9\%). La ganadería familiar está integrada por aves de corral $(70 \%)$, cerdos $(15 \%)$, cabras $(10 \%)$ y en menor proporción ganado bovino $(5 \%)$. Los usos registrados son: alimento ( $80 \%$ ), obsequios y regalos $(15 \%)$, medicinal $(5 \%)$ y el $100 \%$ recupera el excremento como abono. El manejo preferente es en corral tradicional (89\%). La alimentación del ganado está integrada por maíz quebrado, tortilla remojada, desperdicios de verdura y termitas. Las enfermedades frecuentes son viruela y diarreas, las cuales se tratan con remedios caseros. Los conocimientos tradicionales para el manejo del ganado son transmitidos por las mujeres.

Palabras-clave adicionales: manejo, traspatio, unidad familiar, uso.
\end{abstract}

\begin{abstract}
The family livestock, an everyday element of the backyards of the community of Juan Nepomuceno Álvarez, Copala, Guerrero, Mexico) - The aim of this study is to analyze the cultural and economic importance of familial animal husbandry as a rural production system in the families of the community of Juan Nepomuceno Álvarez. Fifty-three questionnaires were applied in family units. The system of production is traditional, with family labor involved, for personal consumption of meat and eggs $(91 \%)$ and for commerce $(9 \%)$. Familial animal husbandry is integrated by poultry $(70 \%)$, pigs $(15 \%)$, goats $(10 \%)$ and a small percentage of cattle $(5 \%)$. The registered uses were: food $(80 \%)$, presents and gifts $(15 \%)$, medicinal $(5 \%)$ and a $100 \%$ recuperates the feces for its use as fertilizer. The management scheme preferred is the traditional pen $(89 \%)$. Feeding includes broken corn, tortillas soaked in water, vegetable scrapes and termites. Frequent illnesses are smallpox and diarrheas, which are treated with home remedies. The traditional knowledge for its management are transmitted by women.
\end{abstract}

Additional key words: backyard, family units, management, use.

Dentro del sector pecuario en México y Latinoamérica, en pequeñas y medianas unidades productivas se distingue a la ganadería doméstica como "ganadería familiar" o de "traspatio" (Sántiz et al. 2012), que en general se desarrolla con prácticas tradicionales. Esta actividad representa una fuente importante de alimentos y bienes para las familias campesinas que la practican. Se basa en el trabajo de todos sus integrantes, principalmente de las mujeres, quienes se encargan de la organización del traspatio en general (Allende et al. 2012) además de otras actividades productivas.

Se concibe que la práctica de la ganadería familiar se enmarca en un complejo de actividades que conforman un ingreso total de la unidad familiar campesina que está conformado por objetivos y decisiones diversas. Se entiende que para garantizar la reproducción social campesina se ejecutan un conjunto de procesos que tienen como resultado la subsistencia de la unidad familiar y del grupo campesino en general. $\mathrm{La}$ reproducción social se entiende desde una perspectiva amplia, que engloba la reproducción

\footnotetext{
*Autora para la correspondencia: elsaguzmang@yahoo.com.mx; agafa666@hotmail.com

Editor correspondiente: Ana H. Ladio

Envio: 12 nov. 2013; acepto: 25 jun. 2014

Publicación electrónica: 12 nov. 2014; versión final: 23 dez. 2014
}

biológica, material, económica, social y cultural del grupo, de manera que los procesos abarcan igualmente aspectos diversos para poder garantizar la reproducción del conjunto de aspectos. Así se lleva a cabo una estrategia de vida o estrategia adaptativa sustentada en la lógica campesina de subsistencia y reproducción social, dentro de la sociedad que contiene al grupo.

La estrategia campesina de vida se concibe como opciones guiadas y valores interiorizados que se toman considerando logros o fines a distintos tiempos, y que en su transcurso se van adecuando y renovando, de acuerdo a factores, posibilidades y limitaciones internas y externas (Guzmán 2005). Estas opciones se encuentran estructuradas por una división social, sexual y técnica del trabajo, que posibilita o limita a los individuos a desarrollar sus capacidades (Oliveira \& Salles 1988).

La estrategia productiva se construye teniendo como base la unidad doméstica o familiar, a través de la cual participan sus integrantes en los procesos socioeconómicos que la sustentan, lo cual implica definir una serie de decisiones a partir de los objetivos planteados en el seno de la unidad familiar, de acuerdo a los recursos disponibles (manos, tiempos, esfuerzos, capacidades, conocimientos, experiencias y espacios) y de las pautas de organización y relaciones para enfrentar la situación de escasez en que subsisten las 
familias campesinas para concretar un sustento, o ingreso total.

La organización de la unidad familiar se concreta con la distribución de tareas de los diferentes integrantes de la misma que desarrollarán diversas actividades para obtener productos o ingresos monetarios que conformarán dicho ingreso total (Salles 1998). Las actividades se organizan en el desarrollo de las tareas para responder a las diferentes necesidades colectivas que se proponen de acuerdo al sexo y a la edad en una estructura jerárquica (Heyning 1992; Cragnolino 2002; Lanza \& Rojas 2010).

La estrategia campesina contempla objetivos y ámbitos de actividades que se complementan entre sí. Ciertas actividades cubren un objetivo básico de garantizar la seguridad de la subsistencia a partir de los recursos y trabajo propio, estos incluyen actividades dentro del ámbito doméstico y el traspatio, así como las actividades agrícolas en la parcela cuyos productos se destinan básicamente al autoconsumo o al intercambio y comercialización en circuitos cortos, como por ejemplo en la misma comunidad o las plazas locales. Igualmente se cuentan con actividades que contemplan el objetivo de vinculación al mercado, para la obtención de ingresos económicos, el cual se integra por un ámbito de producción agrícola comercial y otro de movilidad y venta de fuerza de trabajo en el sector agrícola y en otros sectores económicos, incluyendo procesos migratorios (Guzmán 2005).

En este caso interesa apuntar que en el ámbito de seguridad, en las actividades de traspatio se encuentra la ganadería de traspatio, como una actividad, entre otras que contribuye a garantizar la seguridad campesina como un valor, objetivo y prácticas de la misma forma de vida del grupo. Contribuye a garantizar posibilidades de subsistencia frente a la situación de vulnerabilidad que la pobreza campesina implica, por lo que los productos alimenticios que en este ámbito se generan se vuelven imprescindibles dentro de la gama de productos que la familia campesina genera en el conjunto de procesos productivos.

Las actividades que se llevan a cabo dentro de los espacios domésticos y productivos no comerciales producen recursos básicos para la reproducción campesina, sin el carácter de incertidumbre que la venta de fuerza de trabajo o productos en circuitos comerciales amplios implica, es por esto que se considera que constituyen elementos de seguridad. Las actividades domésticas y las productivas que generan productos para el auto-sustento y autoconsumo, que no requieren pasar por las lógicas comerciales y sostiene experiencias y conocimientos en las familias, así como en las comunidades a través de procesos de intercambios.

Estas actividades de auto-sustento se fundan en el origen mismo de los grupos campesinos y se sostienen por la transmisión de conocimientos para el ejercicio de las prácticas. A través de la estrategia, la familia usa, maneja y reproduce los recursos naturales, además de transmitir en los procesos cotidianos el conocimiento ancestral heredado de padres a hijos, como herramientas tradicionales que les permiten realizar el manejo integrado de sus recursos (suelo, agua, plantas, animales y recursos marinos) presentes en su territorio.

Así, el conocimiento tradicional para el manejo y cría de animales domésticos en las familias se mantiene y recrea en los traspatios, y en lo general garantiza la obtención de productos como huevo, carne, leche $\mathrm{y}$ elaboración de otros como queso, para el autoconsumo de la familia, así como los ingresos económicos de la venta eventual de los productos, o la obtención de otros productos necesarios si es que se da el intercambio interfamiliar.

La cría de animales domésticos como sistema de producción de "ganadería de traspatio" es llevado a cabo principalmente por las mujeres incluyendo la explotación ganadera en pequeña escala, en la cual se crían aves de corral, ganado porcino, caprino y bovino (Román 1989), ya que este ámbito se fusiona a las actividades domésticas y forman parte del panorama de actividades productivas de las mujeres, al menos como organizadores y responsables del traspatio mismo, con la colaboración puntual de otros integrantes de la familia.

Es así que se considera que los traspatios y la ganadería familiar son eslabones imprescindibles para la reproducción campesina. Sin embargo, a pesar de su importancia alimentaria, económica y el significado cultural, actualmente se observa una tendencia a la disminución de dichos espacios productivos, por diversos factores, como el incremento de las actividades terciarias (Aquino et al. 2003), que se dan, en el marco de la pérdida del peso económico, que el sector agropecuario va tomando, como parte de las macro tendencias que las políticas neoliberales marcan, al priorizar la importación de alimentos y el apoyo a actividades productivas de exportación, resultando en abandono de los apoyos gubernamentales generales a la producción agropecuaria, y la necesidad de que las familias campesinas se enfoquen a la obtención de ingresos en los sectores no agrícolas o en la migración.

De esta manera, interesa documentar y resaltar la presencia actual de los procesos agropecuarios comunitarios y familiares que puedan generar información para problematizar las tendencias de disminución, así como las persistencias de las actividades campesinas, en particular de la ganadería familiar. El objetivo del presente trabajo es conocer la importancia cultural y económica de la ganadería familiar como sistema de producción campesina en las familias de la comunidad Juan Nepomuceno Álvarez, del municipio de Copala, Guerrero, México. Se busca documentar los componentes del traspatio y en éste el papel de la ganadería familiar como subsistema dentro de la estrategia general de reproducción campesina, destacando las características de los procesos 
productivos, la organización familiar, los roles de género, así como sus aspectos económicos y culturales.

Características generales de la zona de estudio. Geográficamente, la comunidad Juan Nepomuceno Álvarez (Figura 1) se ubica en la Sierra Madre del Sur (ca. $\left.16^{\circ} 32^{\prime} \mathrm{N}, 98^{\circ} 54^{\prime} \mathrm{W}\right)$, dentro de la región denominada Costa Chica de Guerrero, México; a una altitud de $10 \mathrm{~m}$ s.n.m. (INEGI 2010). Se presenta un clima cálido subhúmedo con lluvias en verano (Aw1), con una temperatura media anual de $26-28^{\circ} \mathrm{C}$. La precipitación media anual oscila entre los 1000-1500 mm (INEGI 2009).

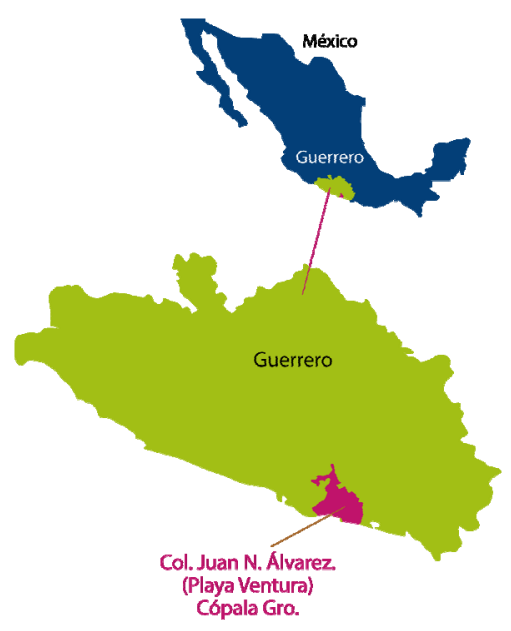

Figura 1. Ubicación de la comunidad Juan Nepomuceno Álvarez, Copala, Guerrero, México.

La comunidad estudiada pertenece a la región hidrológica Costa Chica-Río Verde, de la cuenca Río Nexpa y a las subcuencas Río Copala (67.02\%), Chautengo (16.99\%) y Río Marquelia (15.99\%) (INEGI 2010). Los cuerpos de agua más importantes para la comunidad son la laguna Cabeza de Charco y el río Copala. Además, existen arroyos o apantles como se les conoce localmente, pero estos únicamente abastecen de agua en la época de lluvia a la agricultura y ganadería.

Se presenta agricultura de temporal, de riego y humedad, desarrollando principalmente cultivos de maíz con asociación de frijol, calabaza, jamaica y sandía. En relación a la flora, en la vegetación acuática, están presentes algunas especies abundantes como son el lirio acuático y el tule. La selva baja caducifolia se distribuye principalmente en el Cerro del Coacoyul y se observan relictos en áreas aledañas al pueblo. Ésta se caracteriza por árboles de 8-12 $\mathrm{m}$, que se ramifican cerca del suelo; durante la temporada de secas, la mayor parte de las copas pierden sus hojas (Miranda \& Hernández 1963). Algunas especies representativas de este tipo de vegetación con valor de uso para la comunidad son: la parota Enterolobium cyclocarpum (Jacq.) Griseb., guiro Crescentia alata Kunth, ayoyote Thevetia thevetioides (Kunth) K.Schum., zopilote
Swietenia humilis Zucc., palo de brasil Haematoxylun brasiletto H.Karst., icaco Chrysobalanus icaco L., copal Bursera sp., cubato Acacia sp., guapinola Hymenaea courbaril L., guarumbo Cecropia obtusifolia Bertol., cuachalalate Amphipterygium adstringens (Schltdl.) Standl. El manglar se localiza al oriente de la comunidad conformado por árboles de mangle Rhizophora mangle L., el cual aporta leña que utilizan las familias para cocinar.

\section{MATERIAL Y MÉtodos}

Se realizaron visitas a la comunidad para informar a los pobladores sobre el propósito del trabajo y contar con su apoyo. Posteriormente, se realizaron recorridos con guías locales a las casas de las familias con la presencia de animales de traspatio, bajo los criterios que proponen Aquino et al. (2003), que consiste en la identificación de unidades productivas familiares y que estén dispuestas a colaborar con la investigación. Para recopilar la información sobre el conocimiento tradicional, se aplicaron técnicas etnobiológicas como entrevistas y encuestas, se trabajó con las mujeres de cada traspatio (Barrera 1983; Martín 1997).

Se hicieron entrevistas semiestructuradas con base en un cuestionario previo (Apéndice 1) en el que se obtuvo el nombre común, interés en la crianza, formas de manejo y uso, enfermedades, tratamientos, comercialización y destino de la producción. A las primeras mujeres entrevistadas se les invitó y aceptaron guiar las visitas a otras casas. Se usaron expresiones y palabras comunes lo cual generó confianza. Algunas entrevistas se grabaron, con el consentimiento del informante (Sturtevant 1964; Bellamy 1996; Costa-Neto 2000). Complementariamente el trabajo de campo, se apoyó en las técnicas de observación participante y diálogo dirigido (Rodríguez et al. 2012). La información recopilada se analizó con estadística descriptiva.

\section{RESULTADOS}

Se muestrearon 53 traspatios, donde se aplicaron entrevistas a 51 amas de casa y dos jefes de familia donde no se cuenta con la figura femenina.

Componentes del traspatio. Dentro de la estrategia familiar de la comunidad Juan Nepomuceno Álvarez, las unidades de producción conjuntan una estrategia conformada por la producción del traspatio, la agricultura (milpa y cultivos asociados), ganadería mayor y menor, la pesca ribereña (una gran variedad de peces, así como ostión, caracol, pulpo y langosta), la cacería (iguanas, venados, armadillos, jabalíes para consumo de carne), la recolección de plantas medicinales, frutos $\mathrm{y}$ leña, $\mathrm{y}$ servicios de turismo $\mathrm{y}$ 
ecoturismo (recorridos de observación de recursos naturales, pesca, buceo, kayak).

En especial interesa resaltar el papel del traspatio como ámbito de reproducción de la ganadería familiar, objeto de este estudio. Como elemento de su funcionamiento se encuentra su estructura, es decir el conjunto de componentes para la producción del traspatio. El conjunto de actividades resultan altamente productivas considerando que en su limitada superficie (200 $\mathrm{m}^{2}$ en promedio) hacen constantes $\mathrm{y}$ diversas aportaciones a la unidad doméstica; involucra un trabajo basado en una vasta diversidad, turnando durante el ciclo productivo diversos cultivos tradicionales como el de la milpa-frijol-calabazasandia.

En el traspatio, para el manejo general se crían pequeños grupos de animales domésticos aprovechando el conocimiento tradicional y los recursos disponibles en la unidad de producción. Las contribuciones que el traspatio rinde a la economía doméstica son múltiples y considerables, y éstas se presentan en versiones de ahorro o bien como pequeños ingresos económicos, así como en el aporte básico de productos para la dieta tradicional de la familia, y puede de vez en cuando intercambiar con vecinos y familiares los productos cosechados, o incluso venderlos en las plazas locales de los municipios de Marquelia y Copala, Guerrero, México.

Entre las actividades de traspatio que se desarrollan en la comunidad, la ganadería familiar se integra principalmente por la cría de aves de rancho como gallinas y guajolotes para autoconsumo familiar en la mayoría de los casos. En el establecimiento, cuidado y manejo de los traspatios participan activamente los integrantes de la familia, de esta manera se obtienen frutas, semillas, legumbres, flores, tubérculos, tallos, así como el conjunto de productos de la ganadería. Los productos de los traspatios de la comunidad aportan a la seguridad alimentaria y los ingresos familiares, favoreciendo las oportunidades para mejorar la calidad de vida de las familias, de esta manera se recrean las prácticas y roles familiares para garantizar que las actividades se lleven a cabo y se obtengan los productos de autoconsumo.

Procesos productivos de la ganadería de traspatio. La ganadería familiar en la comunidad en estudio es una actividad popular que se relaciona a la cría, de gallinas, guajolotes, "cochis" (cerdos), cabras y en menor proporción vacas. El 94\% de las mujeres entrevistadas se encargan de la cría y manejo de los animales y poco frecuente del ganado bovino, que es una actividad en la que el jefe de familia lleva acabo, especialmente en el pastoreo que traspasa los límites del traspatio, aunque son ellas quienes dirigen el trabajo en general.

La ganadería familiar de la comunidad está integrada por 580 aves de corral gallinas (Gallus gallus L., 1758) y 350 guajolotes (Meleagris gallopavo L.,
1758), 105 cerdos (Sus scrofa L., 1758), 40 cabras (Capra aegagrus hircus L., 1758) y 25 vacas (Bos taurus L., 1758) criados por 51 mujeres y 2 varones (jefes de familia) de la comunidad. Existe la preferencia por tener más animales hembras (gallinas, pípilas, cochis o vacas), lo cual se debe, a mención principalmente de las mujeres entrevistadas, por el aporte de carne, leche y huevos, los que son utilizados como alimento. La cría de animales de traspatio representa además una caja de ahorro, en virtud de que en situaciones difíciles venden a los animales o crías para hacer frente a algunos problemas como enfermedades de la familia, la muerte de un familiar o para adquirir útiles escolares de sus hijos.

Respecto al manejo, se considera del tipo tradicional, ya que no existen elementos tecnológicos orientados a la alimentación de los animales, manejo de enfermedades, genética o reproducción y comercialización. El corral tradicional (89\%) es la forma preferida por las mujeres para mantener encerrados a los animales, mismos que son construidos por el jefe de familia con hoja de palma y palos de diferentes plantas del monte (quebrache, palma, zopilote, parota y huizache). Este consta de un área aproximada de $15 \mathrm{~m} 2$ con una puerta de acceso que permite el paso a los miembros de la unidad familiar para llevar a cabo diferentes labores. Dentro del corral las mujeres habilitan espacios construidos con materiales de la región (bejucos) para que los animales descansen y "Chitas" para que las gallinas y pípilas depositen sus huevos y los empollen. Otros materiales complementarios al corral son la tela de gallinero (11\%), para el techo se utilizan enramadas construidas con ramas frescas y secas de palma. Es común también observar que anden sueltos los animales en el traspatio aledaño a la casa donde se alimentan de yerbas silvestres y organismos como los insectos. Cuando cae la tarde, las mujeres localizan y arrean a las gallinas, pípilas, guajolotes, "cochis" y crías a la casa para su resguardo en el corral. Para evitar la confusión de animales en cuanto a la propiedad, se les amarra un hilo de color en la pata o cresta.

La alimentación de los animales es tradicional, está integrada por grano de maíz quebrado o entero (Zea mays L.) o tortilla remojada, desperdicios de la casa principalmente verdura y termitas para las aves de corral. El uso de alimentos balanceados no se presenta ya que, por ejemplo, una señora señalo su elevado precio, además alimentando a los animales con comida natural los habitantes de las comunidades como el Papayo, Las Salinas y Marquelia de la Costa Chica de Guerrero recurren a la comunidad para comprar gallinas, pipilas, guajolotes o cerdos, porque en su opinión la carne es más limpia y sabrosa. Otra forma de alimentarlos es por el pastoreo de plantas silvestres e invertebrados que se localizan en los traspatios o áreas aledañas a la casa. Esto significa que es un sistema que no permite la inversión de dinero para su sostenimiento, y que la posibilidad de la venta está 
basada en el reconocimiento de la salud del producto al ser alimentado de manera natural.

Se reportan para las aves de corral dos tipos de enfermedades frecuentes en la época de lluvias: la viruela $(75 \%)$ y diarreas $(25 \%)$. En la época de frío, se reportan catarro y gripa. Es evidente la nula vacunación y desparasitación. El tratamiento de las enfermedades se realiza mediante la utilización de remedios caseros $(91 \%)$, untan tomate asado, limón o "lodo" que extraen de la laguna Cabeza de Charco" en la parte afectada o cuerpo del animal para curar la viruela. Para combatir la diarrea o parásitos intestinales, se les da de tomar agua de coco (Cocos nucifera L.) o con cal. Solo aquellos productores (9\%) que destinan su producción exclusivamente a la venta utilizan vacunas o pagan un veterinario. Cabe señalar que algunas mujeres $(n=4)$ mencionaron colocar un pedazo de carne de víbora de cascabel (Crotalus sp.) en agua, misma que posterior dan a beber a los animales. De acuerdo a las mujeres, esto sirve para que los animales no se enfermen. Otras explicaron que es mejor dejarlos andar libres porque al comer plantas silvestres los protege contra enfermedades. Una informante mencionó que deja libres a sus animales en la época de lluvias para que se alimenten de ajolotes, lo cual también permite que no se enfermen. Además, se utiliza cal o ceniza que se dispersa en los corrales para contrarrestar las enfermedades y contagios de otros animales. Cuando hay animales enfermos se sacan del corral y se amarran hasta que se curan.

Organización familiar y roles de género. El sistema de producción de ganadería familiar identificado con base en la FAO (2002) en la comunidad es de tipo tradicional, desarrollado por las unidades familiares en los traspatios de la casahabitación, en la que sus integrantes (jefe de familia, mujer, hijos, nietos y nueras) aportan el trabajo. Se utilizan poco insumos y los productos se destinan al autoconsumo. Este sistema se vio asociado a una organización familiar en la que las mujeres son las encargadas de la organización y responsables de los trabajos del traspatio, en vistas de que los varones trabajan prioritariamente en las parcelas agrícolas.

En su jornada de trabajo, la mujer combina las tareas de atención al esposo e hijos, cuidado de la casa $\mathrm{y}$ del traspatio, saca del gallinero las aves para que tomen baños de sol y busquen insectos o pasto para comer, libera a los "cochis" (cerdos), cabras y vacas del corral y los acompaña a pastorear unas horas al monte o a las áreas cercanas a la laguna llamada localmente "cabeza de charco"; mientras las cabras y vacas se alimentan, ellas aprovechan para recolectar plantas medicinales, frutos o leña que posteriormente servirán para preparar el fuego de la cocina o bien pescan en la laguna organismos como charras, cuatetes, lisas, camarón, almejas, etc. Por la tarde, limpia sus plantas, remueve la tierra, limpia los corrales de sus animales (gallinas, guajolotes, cerdos, cabras y vacas) y cosechan algunos productos como chiles, coco, epazote, mango, maracuyá etc. Las mujeres comparten productos como cocos, plantas medicinales, huevos, pollos o pípilos o fruta de temporada como mango, nanches y plátano. Las tareas también incluyen la selección de los alimentos para los animales, su curación con remedios caseros, dirigen la construcción de corrales así como la venta de animales.

En este espacio propio, las mujeres, deciden, recrean, eligen y realizan actividades con base a sus gustos y preferencias en beneficio de la familia. Saben que el traspatio es su alternativa de apoyo para los tiempos de crisis económica y alimentaria y pueden disponer de sus recursos, sin tener que pedir autorización ni ayuda. Los conocimientos tradicionales generados son transmitidos de una generación a otra, $\mathrm{y}$ la enseñanza y aprendizaje son continuos. Por esto, se considera que el papel de la mujer es clave en el desarrollo y preservación de esta actividad. Su participación en el manejo de los animales es mayor en comparación con los demás miembros de la unidad familiar. Además, se encargan de otros trabajos en el traspatio, así como su organización general y funcionamiento; en total, se calcula que ellas destinan a las labores con los animales de 30 minutos a 2 horas en función de la actividad, y los varones ayudan eventualmente.

Es evidente la función de la mujer en las labores referentes a la cría y manejo de la ganadería familiar, $74 \%$ de éstas, organizan los alimentos que se les darán a los animales, mientras que un $15 \%$ de hombres apoyan con el transporte de alimento proveniente de la parcela. El manejo de enfermedades y la preparación de los remedios caseros para curarlas, así como la venta de los animales o sus productos como el huevo son actividades propias de la ama de casa. La limpieza de los corrales se divide entre las amas de casa (80\%), los hijos $(10 \%)$ y nueras $(10 \%)$. El jefe de familia construye los gallineros y corrales $(80 \%)$ o encierros para los animales, sin embargo un $20 \%$ de las mujeres apoyan en la construcción, seleccionan el espacio y los materiales a utilizar. Los hijos y nueras también participan en las diferentes labores pero en menor proporción. Por ejemplo, apoyan en dar de comer o poner agua a los animales y a colectar los huevos a diario. Además, las mujeres $(100 \%)$ son quienes supervisan y ponen en cuarentena a los animales enfermos. Cabe mencionar que las mujeres en todo momento supervisan las labores que realizan cada uno de los miembros de las familias.

El trabajo del traspatio en la comunidad Juan Nepomuceno Álvarez se divide entre los miembros de la familia, en donde la mujer, además de otras tareas asignadas a su género como la limpieza de la casa, la preparación de los alimentos y el cuidado de los hijos. Es responsable de la organización y funcionamiento general del traspatio, lo que se traduce en un ámbito de confianza y decisión, en la realización de tareas productivas, venta de productos como huevos, 
animales, y en el intercambio de animales o plantas con otras mujeres de la misma comunidad o región.

Dada su actividad en el ámbito doméstico, las mujeres tienen un papel fundamental en la transmisión de conocimientos a las generaciones jóvenes para mantener vigente la cría de animales, principalmente para el sustento familiar. También, las prácticas de cría de animales en el traspatio se recrean en los intercambios de experiencias y animales inter y extracomunidad.

Para las mujeres, las razones por las que se mantiene vigente la cría de las gallinas y guajolotes principalmente y otros animales son: aporte de carne y huevos a la alimentación familiar $(89 \%)$, por tradición familiar $(65 \%)$, por la venta $(9 \%)$, por ser obsequios para las fiestas de la comunidad (10\%).

Algunas mujeres $(\mathrm{n}=35)$, expresaron que anteriormente la cría de otros animales de corral como los puercos, cabras y vacas era más abundante, pero debido a la falta de espacio en la casa se han ido eliminando. Otras causas son la falta de interés por las generaciones jóvenes, los cuales mencionan que requieren muchos cuidados y que es mejor dedicarse al turismo o a la pesca.

Destino de los productos de la ganadería de traspatio. En la comunidad en estudio se registraron dos tipos de unidades de producción de ganadería familiar de acuerdo al destino de la cría: 1- unidades de autoconsumo (91\%), que son aquellos que destinan sus animales para el sustento de la familia, y que ven en los animales de traspatio una caja de ahorro, ya que sólo se venden cuando se tiene alguna necesidad o como ellos llaman "urgencia" y 2- unidades comerciales (9\%), que destinan su producción exclusivamente para la venta.

La importancia económica de la ganadería de traspatio se encuentra en la venta de los animales o sus productos en el mercado local o en las cabeceras municipales de Copala y Marquelia en la Costa Chica de Guerrero, que realizan las unidades comerciales, pero igualmente, en menor medida las unidades de autoconsumo. Igualmente, existe interés económico por las familias que crían animales de traspatio en virtud de su rendimiento en carne, bajo costo de producción y la calidad nutritiva de algunas especies como el guajolote, aunado a su bajo contenido de grasa en la carne (BSTID 1991; Castellanos 2004), características que se valoran tanto en los usos comerciales, como en el destino preponderante que es el de autoconsumo. Son tres las mujeres que crían aves como los guajolotes exclusivamente para la venta y son ellas las encargadas de su comercialización en el hogar, los restaurantes locales donde se les ofrecen a los turistas como un platillo tradicional o en los mercados de Marquelia y Copala en la Costa Chica de Guerrero. Los productos que son destinados para la venta son los huevos, con un costo por unidad de \$ 2.00 a \$ 5.00, o bien los animales juveniles y adultos de Gallus gallus (gallina) se venden en $\$ 30$ a $\$ 60$; el animal adulto de
Meleagris gallopavo (guajolote) vivo se vende en $\$$ 150 en temporada baja y de $\$ 250$ a $\$ 350$, dependiendo de la época y ocasión, como en bautizos, bodas $y$ en diciembre que es cuando arriban a la comunidad turistas para festejar la navidad.

La contribución de la ganadería familiar al ingreso, es a partir de la función de ahorro que tiene la crianza de animales criollos para solventar algunas necesidades básicas de la familia, al venderlos o intercambiarlos. La ganadería de traspatio tiene como objetivo crear un fondo que permita a la familia subsistir en épocas de crisis, asegurando el mínimo básico de subsistencia (calorías) para reponer el gasto de energía empleado en el trabajo. Además representa una fuente de ahorro y ganancia económica para situaciones de emergencia.

Mujeres provenientes de otros pueblos arriban a la comunidad, de las Salinas se intercambia sal de mar, del Papayo maíz o frijol, sandías, papayas, y de Marquelia llevan pescado para poder intercambiarlos por animales del traspatio. Por ejemplo, las pípilas de M. gallopavo se ponen a la venta después de por lo menos tres periodos de reproducción ("puestas"), es decir una vez que han permanecido de 1 a 3 años en la unidad familiar, para aprovechar su capacidad reproductiva y mantener el sistema vigente.

Los usos registrados que la población le da a los productos de la ganadería son: alimento (80\%), obsequios y regalos (15\%), medicinal (5\%) y el $100 \%$ mencionó el uso del excremento como abono. El uso alimentario se refiere al consumo de huevos y carne, se prepara en mole rojo o mole de pipián, asado con chile ajo, en caldo o frito. El uso como obsequio, presenta tres destinos: 1- en fiestas del pueblo, se acostumbra a regalar animales de traspatio; 2 - cuando se visita a los compadres se les lleva mole de guajolote; 3- en los cumpleaños, bodas o bautizos se preparan diferentes guisos. Para el uso medicinal, se utilizan los huevos o los animales vivos para curar algunas enfermedades culturales, como el "aire", el "daño" o "mal de ojo", principalmente a los niños. Consiste en tomar un huevo o el animal y frotar sobre el niño con el padecimiento, pidiendo a "Dios" su cura. En todos los traspatios, es común el uso del excremento de gallinas, guajolote, cabras y vacas como abono para los cultivos o plantas comestibles y medicinales presentes en los traspatios.

\section{DISCUSIÓN}

En las investigaciones y reflexiones sobre las estrategias de reproducción social, se reconoce en la literatura la adaptación de los grupos a las condiciones circundantes, locales, como a las dinámicas y cambios de la sociedad misma, como lo mencionan Lehalleur \& Rendón (1989). Este mecanismo es el que consideramos que las familias de la comunidad Juan Álvarez Nepomuceno están llevando a cabo para garantizar su subsistencia en las condiciones cambiantes del medio rural y la presión de tendencias 
como la migración. Así lo vemos igualmente en casos de Oaxaca (Sánchez 1995) y Puebla (Ramírez 2008).

La ganadería de traspatio es una actividad ancestral como se menciona para el caso de las culturas indígenas mexicanas en la domesticación y uso del guajolote (Meleagris gallopavo) que se remota a más de 7000 años cuando comenzó el proceso de domesticación (Valadez 2003). El gran arraigo de la ganadería de traspatio en comunidades campesinas se reconoce en otros países de América Latina, en Asia y África, y su importancia radica en tanto abastece necesidades sociales, religiosas, el aporte de carne y huevos (Sonaiya et al. 2002). Básicamente, se ha reportado, como el caso de este estudio, que constituye un importante aporte alimentario e ingreso económico (Medrano 2000).

De esta manera, se reconoce que la ganadería de traspatio, como integrante del traspatio y de las estrategias de reproducción campesinas, abarca valores y reconocimientos más allá de ser meramente una actividad productiva, pues así como se vio que es parte de las actividades de vida de las familias campesinas de Guerrero; en la documentaciones, se encuentra el reconocimiento del traspatio como espacios de vida distribuidos en las áreas rurales de América Latina donde se aprovecha el área física circundante a la vivienda donde habita, produce y se reproduce la familia campesina (Jerez 1994; Estrada et al. 1998; Lok 1998; Steinberg 1998; Lerner et al. 2009). La importancia de los traspatios igualmente se coloca en que éste se integra de conocimientos locales aplicados a la producción de alimentos, en el uso y manejo de los recursos naturales (Delgado 2008).

Se ha demostrado que parte de la lógica del traspatio radica en la organización del trabajo, la cual se basa claramente en el trabajo de las mujeres. Esto representa un valor cultural campesino, dado que es una característica encontrada en otros estudios. Hernández \& Jaimes (2003), Vieyra et al. (2004) y Losada et al. (2006) también mencionan que la atención de los animales de traspatio es una actividad desempeñada principalmente por las mujeres, resaltan la importancia de ella en el mantenimiento de los traspatios y su trascendencia en la distribución del trabajo doméstico. Es decir, se trata de un trabajo constante, aunque en casos no de mucha demanda, que realizan junto a otras actividades de la casa y traspatio.

Estos resultados coinciden con otros estudios realizados con productores de Xoxocotla, Morelos (García et al. 2012), Dzununcan, Yucatán (Rodríguez et al. 1996), Texán y Tzacala en Mérida, Yucatán (Rejón et al. 1996).

Las prácticas de la ganadería de traspatio son sostenida por conocimientos empíricos que se constituyen como tradiciones que se comparten (Soler 2010). En lo general, en otros estudios, se caracterizan como una práctica de pocos insumos. Así en cuanto al manejo de las enfermedades de animales con hierbas, sin medicación ni vacunas, son reportadas por Jiménez et al. (2012). López et al. (2008) también las reportan para la región del Balsas y García et al. (2012) para Morelos, Michoacán (López et al. 2008), Oaxaca, (Bautista 2006) y Puebla (Hernández et al. 2005), encontrando como principales enfermedades viruela y diarreas en las diferentes regiones.

La alimentación se basa en ingredientes locales como maíz, semilla de calabaza, cacahuate y fruta de mango (García et al. 2012), tortilla remojada y frutos de la región (López et al. 2008).

Se establecen vínculos especiales con los animales, tales que se ha encontrado en distintas regiones que se usan hilitos de identificación en la región Mixe de Oaxaca y se llama a los animales con silbidos que reconocen, comenta Bautista (2006).

Los usos de los productos de los traspatios son principalmente alimentarios (Rejón et al. 1996; Lafòn 1997; Juan \& Madrigal 2005; Bautista 2006; Camacho et al. 2006; Losada et al. 2006; García et al. 2012), con lo que podríamos decir que este es el sentido básico del espacio productivo-doméstico, es decir garantiza el acceso a alimentos producidos en base al propio trabajo $\mathrm{y}$ recursos de la familia campesina.

Llamas (2005) señala la importancia del guajolote en la alimentación familiar porque aporta más proteínas que el cerdo y el pollo, menos grasas que el pollo, res, cerdo, codorniz y conejo y más calorías que el pollo, res, conejo y codorniz. Además es rico en vitaminas B1, B3, B5, B6 y B12, biotina, acido fólico, fosforo, hierro y zinc. Lo anterior, permite reforzar lo planteado por Gueye (2003) y García et al. (2012) respecto a que la ganadería de traspatio en comunidades rurales es un valioso recurso por contribuir a mitigar la falta de recursos alimenticios y la disminución de la pobreza, mediante seguridad alimentaria de las familias campesinas. Por supuesto que alrededor de la alimentación campesina se generan otros procesos relacionales, como los intercambios con vecinos y familiares, los productos cosechados, o la participación en las plazas locales (Rodríguez 2006; Rodríguez et al. 2010). Esto habla que la trascendencia de la ganadería familiar va más allá de las fronteras de los traspatios mismos.

Ciertamente, se ha reportado en distintos casos los problemas actuales que las comunidades y traspatios campesinos viven, que llevan a la distinción de tendencias de disminución de los traspatios. Así, Aquino et al. (2003) reporta para el estado de Veracruz causas como las enfermedades y/o depredación no consumo de carne y la falta de cerco como causas internas que impactan el desarrollo de los traspatios. Pero también se remarcan causas externas. Sin embargo, persiste la cría de animales de traspatio como sistema de ganadería familiar o de traspatio.

\section{CONCLuSiones}

La cría y manejo de la ganadería familiar como componente de los traspatios y estrategia familiar en la comunidad Juan Nepomuceno Álvarez es una actividad 
productiva tradicional permanente y persistente. Proporciona alimentos cotidianos a la familia, además de generar algunos ingresos monetarios para completar las necesidades de consumo de la familia. La cría de animales de traspatio, bajo el sistema en que se lleva a cabo, no representa un costo económico significativo, pero sí implica la recreación de conocimientos para poder ser sostenida dentro de las actividades de la estrategia general campesina, y representa un peso, en tanto aporte económico y cultural, a la reproducción social del grupo.

La mínima inversión económica y la utilización de recursos de la región para el funcionamiento de los traspatios refuerzan la idea que éste se incluye en el ámbito de búsqueda de seguridad, la cual en condiciones de escasez económica requiere la optimización de recursos. Al mismo tiempo, los productos del traspatio y en particular de la ganadería se encaminan al reforzamiento de dicha seguridad. Sin embargo, este elemento fundamental de la subsistencia campesina, la seguridad, se pone en cuestión en el momento de reconocer las tendencias a la disminución de los traspatios y con ello de la ganadería de este tipo.

Se observan nodos problemáticos en el manejo tradicional de los animales, así que las deficiencias en el manejo de los animales, como en el caso de las enfermedades, representa un elemento de vulnerabilidad. En el marco del contexto actual el estudio de los traspatios, se vuelve necesario reforzar los aspectos internos, para disminuir el balance desfavorable con respecto a las influencias externas, como las preponderancias del sector terciario.

Sostener los niveles de producción, no sólo de cría de animales sino del conjunto de producción del traspatio, a pesar de la inclusión de actividades terciarias o turísticas, ayudaría a mantener el equilibrio de las estrategias como complejos diversos de objetivos; pues el debilitamiento de algunos de sus elementos y la disminución de la diversidad puede atentar con la subsistencia campesina, no sólo de la cría de animales.

Se reconoce que es necesario reforzar el sostenimiento de las prácticas campesinas de traspatio, y en particular del trabajo que se invierte en éstas. Así, la valoración del trabajo de la mujer es fundamental, en especial en relación a las opciones laborales del conjunto de la población y a las tendencias sectoriales. La unidad familiar es la base de la estrategia y en este caso la participación de la mujer básica para la ganadería de traspatio, y el sostenimiento del traspatio como subsistema. Otro elemento que es necesario resaltar y valorar es el papel de los intercambios y las redes de intercambio que por un lado, fortalecen la alimentación y seguridad de las familias, y por otro recrean las prácticas productivas. De esta manera, la persistencia de la ganadería de traspatio y la recreación de la estrategia campesina se encuentran asociadas a la optimización de las prácticas productivas, la persistencia del trabajo de la mujer, el sostenimiento de las redes relacionales entre las familias en el intercambio de productos del traspatio, y a la recreación y transmisión de conocimientos a las nuevas generaciones. Esto fortalecería la estrategia y compensaría las problemáticas actuales de los traspatios, sosteniendo, a su vez, a la estrategia y persistencia campesina.

\section{AGRADECIMIENTOS}

A las mujeres de la comunidad Juan Nepomuceno Álvarez, quienes aportaron no sólo su conocimiento y su valioso tiempo, sino también su amistad. Agradezco en especial a la señora Luz Argüello y Reina Pérez, quienes fueron mis guías en la comunidad. Al Dr. José Manuel Pino Moreno, del Instituto de Biología de la Universidad Nacional Autónoma de México, por su revisión y sugerencias, lo cual enriquecieron el presente trabajo. Al Consejo Nacional de Ciencia y Tecnología de México por la beca otorgada para realizar los estudios de doctorado del primer autor.

\section{REFERÊNCIAS}

Allende, N.R.; Jerez, S.M.P.; Vázquez, D.M.A. \& Villegas, A.Y. 2012. Estudio Etno-ornitológico Auuk en Zompantle, Asunción Cacalotpec, Oaxaca: las aves de traspatio. In: D.M.A. Vázquez \& A.D.G. Lope (eds), Aves y Huertos de México. Instituto Tecnológico del Valle de Oaxaca, Oaxaca, p. 48-49.

Aquino, R.; Arroyo, A.L.; Torres, H.G.; Riestra, D.D.; Gallardo, L.F. \& López, B.A. 2003. El guajolote criollo (Meleagris gallopavo L.) y la ganadería familiar en la zona centro del Estado de Veracruz. Técnica Pecuaria en México 41(2): 165-173.

Barrera, A. 1983. Perspectivas para el Quehacer Etnobotánico en México. In: A. Barrera (ed.), La Etnobotánica: tres puntos de vista y una perspectiva. Instituto Nacional de Investigaciones sobre Recursos Bióticos, Xalapa, p. 25-28.

Bautista, J.M. 2006. Diseño de una estrategia de transferencia de tecnología en la ganadería campesina de la región Mixe (Ayuuk) en Oaxaca, México. Raximhai 2(2): 419-433.

Bellamy, R. 1996. Ethnobiology in Tropical Forests. Expedition Advisory Centre, London, p. 18-35.

BSTID (Board of Science and Technology for International Development) 1991. Microlivestock: little-known small animals with promising economic future. National Academies Press, Washington, p. 157-166.

Camacho, E.M.A.; Lira, T.I.; Ramírez, C.L.; López, P.R. \& Arcos, G.J.L. 2006. La avicultura de traspatio en la costa de Oaxaca México. Ciencia y Mar 10(28): 3-11.

Castellanos, E.I. 2004. Punto de acuerdo en relación a la importación de carne de pavo a México. Gaceta del Senado de la República 85. México D.F.

Costa-Neto, E. 2000. Conhecimento e uso tradicional de recursos faunísticos por una comunidade afro-brasileira. Resultados preliminares. Interciencia 25(9): 423-431.

Cragnolino, E. 2002. La unidad doméstica en una investigación de antropología educativa. Congreso Argentino de Antropología 
Social. Universidad Nacional de Cordoba. Disponible en http://www.naya.org.ar/congresos/contenido/laplata/LP5/16.htm; consultada el 15 Jun. 2010.

Delgado, M.J. 2008. Persistencia, conocimiento local y estrategias de vida en sociedades campesinas. Revista de Estudios Sociales 29: 122-133.

Estrada, L.E.; Bello, E. \& Serralta, L. 1998. Dimensiones de la etnobotánica: el solar maya como espacio social. In: S.J.A. Cuevas (ed.), Lecturas en Etnobotánica. Publicaciones del programa Nacional de Etnobotánica. Serie Didáctica sobre etnobotánica, No. 1. Universidad Autónoma Chapingo, Estado de México, p. 457-474.

FAO (Food and Agriculture Organization) 2002. Avicultura Familiar. Disponible en www.fao.org; consultada el 28 Sep. 2010.

García, F.A.; Colin, B.H. \& Monroy, R. 2012. Uso y manejo del guajolote Meleagris gallopavo en la comunidad de Xoxocotla, Morelos. Mesoamericana 16(1): 9-17.

Gueye, E.F. 2003. Information dissemination for family poultry research and development. Livestock Research for Rural Development 15(2): 1-10.

Guzmán, G.E. 2005. Resistencia, Permanencia y Cambio: estrategias campesinas de vida en el poniente de Morelos. Universidad Autónoma del Estado de Morelos-Plaza y Valdes Editoriales, Cuernavaca.

Hernández, J.S.; Oviedo, M.R.; Martínez, A.S.; Carreón, L.L.; Reséndiz, M.R.; Romero, B.J.; Ríos, M.J.; Zamitiz, G.J. \& Vargas, S. 2005. Situación del guajolote común en la comunidad de Santa Ursula (Puebla, México). In: Memorias del VI Simposio Iberoamericano sobre Conservación y Utilización de Recursos Zoogenéticos. San Cristóbal de las Casas, p. 277-281.

Hernández, P.J.O \& Jaimes, P.P.X. 2003. La Participación de las Mujeres en el Manejo Integral del Traspatio. Gobierno del estado de Chiapas, Instituto de la Mujer, Chiapas.

Heyning, K. 1992. Principales Enfoques sobre Economía Campesina. Comisión Económica para América Latina y el Caribe (CEPAL).

INEGI (Instituto Nacional de Estadística Geografía e Informática) 2009. Prontuario de Información Geográfica Municipal en los Estado Unidos Mexicanos. Copala, México.

INEGI (Instituto Nacional de Estadística Geografía e Informática) 2010. Anuario Estadístico del Estado de Guerrero. México.

Jerez, S.M. 1994. La gallina criolla en los valles centrales de Oaxaca. In: S.M.P. Jerez, J.H. Herrera \& M.A. Vázquez (eds), La Gallina Criolla en los Valles Centrales de Oaxaca. Reporte de investigación No.1. ITA de Oaxaca-Centro de Investigación y Graduados Agropecuarios, Oaxaca, p. 23-84.

Jiménez, D.J.E.; Vázquez, D.M.A.; Naranjo, P.E. \& Jérez, S.M.P. 2012. Etno Ornitología maya de Lacanjá-Chansayab, Chiapas. In: D.M.A. Vázquez \& A.D.G. Lope (eds), Aves y Huertos de México. Instituto Tecnológico del Valle de Oaxaca, Oaxaca, p. 28-29.

Juan, P.J.I. \& Madrigal, U.D. 2005. Huertos, diversidad y alimentación en una zona de transición ecológica del estado de México. Ciencia Ergo Sum 1281: 54-63.

Lafòn, A. 1997. Distribution, Habitat Use and Ecology of Gould's Turkey in Chihuahua, Mexico. Tesis de Doctorado en Filosofia. Las Cruces New México.
Lanza, V.C. \& Rojas, M.J. 2010. Estrategias de reproducción de las unidades domésticas campesinas de Jucuapa Centro, Nicaragua. Agricultura, Sociedad y Desarrollo 7(2): 169-187.

Lehalleur, M.P. \& Rendón, T. 1989. Reflexiones a partir de una investigación sobre grupos domésticos campesinos y sus estrategias de reproducción. In: O.D. Oliveira, M.P. Lehalleur \& S.V. Almeida (eds), Grupos Domésticos y Reproducción Cotidiana. El Colegio de México, Universidad Nacional Autónoma de México, Editorial Porrua, México D.F.

Lerner, M.T.; Mariaca, M.R.; Salvatierra, I.B.; González, J.A. \& Wall, K.E. 2009. Aporte de alimentos del huerto familiar a la economía campesina Ch`ol, Suclumpò, Chiapas, México. Etnobiología 7: 30-44.

Llamas, J.M. 2005. El guajolote. Asociación Nacional de Tiendas de Autoservicio y Departamentales Asociación Civil, México D.F.

Lok, R. 1998. Introducción a los Huertos Caseros Tradicionales Tropicales. Módulo de enseñanza agroforestal, $\mathrm{N}^{\circ} 3$. Centro Agronómico Tropical de Investigación y Enseñanza, Costa Rica.

López, Z.R.; Cano, C.H.; Monterrubio, R.T.C.; Chassin, N.O.; Aguilera, R.U. \& Zavala, P.M.G. 2008. Características morfológicas y de producción de guajolotes (Meleagris gallopavo) criados en sistema de traspatio en el Estado de Michoacán, México. Livestock Research for Rural Development 20(5): http://www.lrrd.org/lrrd20/5/lope20068.htm.

Losada, H.; Rivera, J.; Cortes, J.; Castillo, A.; González, R.O. \& Herrera, J. 2006. Un análisis de sistemas de producción de guajolotes (Meleagris gallipavo) en el espacio suburbano de la delegación Xochimilco al sur de la Ciudad de México. Livestock Research for Rural Development 18: http://www.lrrd.org/lrrd18/ 4/losa18052.htm.

Martin, G.1997. Ethnobotany People and Plants Conservation Manual. Chapman Hall, London.

Medrano, J.A. 2000. Recursos animales locales del centro de México. Archivos de Zootecnia 49: 385-390.

Miranda, F. \& Hernández, X. 1963. Los tipos de vegetación de México y su clasificación. In: Xolocotzia: Obras de Efraín Hernández Xolocotzi. Revista Geográfica Agrícola. Universidad Autónoma Chapingo, Chapingo, p. 41-53.

Oliveira, O. \& Salles, V. 1988. Reflexiones teóricas para el estudio de la reproducción de la fuerza de trabajo. Argumentos 4: 19-43.

Ramírez, J. 2008. Ruralidad y estrategias de reproducción campesina en el Valle de Puebla, México. Cuadernos de Desarrollo Rural 5(60): 36-60.

Rejón, M.J.; Dajer, A.F. \& Honhold. 1996. Diagnostico comparativo de la ganadería de traspatio en las comunidades Texàn y Tzacala de la zona henequera del estado de Yucatán. Veterinaria México 27(1): 49-55.

Rodríguez, G.G. 2006. Análisis del Sistema de Producción Agropecuario en Colonias Indigenas de San Cristóbal. Tesis de Maestría en Agroecología Tropical. Facultad de Ciencias Agropecuarias, Universidad Autónoma de Chiapas.

Rodríguez, G.G.; Zaragoza, M.L. \& Perezgrovas, G.R. 2010. Los valores disimulados de la producción animal de traspatio. In: Memorias. XI Simposio Iberoamericano sobre Utilización y Conservación de Recursos Zoogenéticos Locales. João Pessoa, p. 36-39.

Rodríguez, G.G.; Sanabria, N.; Ramírez, D.C.; Zaragoza, M.L. \& Perezgrovas, G.R. 2012. La gallina de rancho, elemento cotidiano del sistema de vida en la familia rural en la Frailesca 
Chiapaneca. In: D.M.A. Vázquez \& A.D.G. Lope (eds), Aves y Huertos de México. Instituto Tecnológico del Valle de Oaxaca, Oaxaca, p. 36-37.

Rodríguez, J.C.; Allaway, C.E.; Wassink, G.J.; Segura, J.C. \& Rivera, T. 1996. Estudio de la avicultura de traspatio en el municipio de Dzununcàn, Yucatán. Veterinaria México 27(3): 215-219.

Román, P.H. 1989. Fauna doméstica. In: V.R. Mosqueda, R.O. Ruiz \& R.C. Ávila (orgs), Memorias del Coloquio Retrospectiva y Perspectivas de la Investigación en el Uso de los Recursos Naturales del Trópico Mexicano en Tepetates Veracruz. Centro Regional de Enseñanzas, Capacitación e Investigación para el Desarrollo Agropecuario del Trópico Húmedo y Colegio de Posgraduados, Veracruz, p. 59-82.

Salles, V. 1998. Sobre los grupos domésticos y las familias campesinas: algo de teoría y de método. In: M. Tarrío \& L. Concheiro (coords), La Sociedad Frente al Mercado. La Jornada Ediciones/Universidad Autónoma MetropolitanaXochimilco, México, p. 273-301.

Sánchez, M.J. 1995. Actividades económicas y estrategias de reproducción entre comunidades hablantes de zapoteco en los Valles de Oaxaca. In: C.G. Hubert (Coord.), Globalización, Deterioro Ambiental y Reorganización Social en el Campo. Universidad Nacional Autónoma de México, Instituto de Investigaciones Sociales y Juan Pablos, México D.F.

Sántiz, R.G.; Perezgrovas, G.R.; Rodríguez, G.G. \& Zaragoza, M.L. 2012. Importancia socioeconómica y cultural de las gallinas locales en una comunidad Tsotsil de Chiapas. In: D.M.A. Vázquez \& A.D.G. Lope (eds), Aves y Huertos de México. Instituto Tecnológico del Valle de Oaxaca, Oaxaca, p. 34-35.

Soler, F. D. M. 2010. Importancia de los sistemas avícolas campesinos (pollo de engorde y gallina ponedora) dentro de la unidad productiva y su aporte a la seguridad alimentaria: Estudio de caso Vereda La Pradera, municipio de Duitama, Boyaca. Tesis de Maestría en Desarrollo Rural. Facultad de Estudios Ambientales y Rurales, Pontifica Universidad Javeriana.

Sonaiya, E.B.; Branckaert, R.D.S. \& Gueye, E.F. 2002. Research and Development Options for Family Poultry. Disponible en http://www.fao.org/ag/againfo/subjects/en/infpd/econf_scope.ht ml; consultada el 12 Jul. 2010.

Steinberg, M.K. 1998. Neotropical kitchen gardens as a potential research landscape for conservation biologist. Conservation Biology 12(5): 1150-1153.

Sturtevant, W.C. 1964. Studies in ethnoscience. American Anthropologist 66(30): 99-131.

Valadez, R.A. 2003. La Domesticación Animal. Editorial Plaza y Valdez, México D.F.

Vieyra, J.; Castillo, A.; Losada, H.; Cortes, J.; Alonso, G.; Ruiz, T.; Hernández, P.; Zamora, A. \& Acevedo, A. 2004. La participación de la mujer en la producción de traspatio y sus beneficios tangibles e intangibles. Cuadernos de Desarrollo Rural 53: 9-23. 
Apéndice 1. Cuestionario aplicado a las familias de la comunidad Juan Nepomuceno Álvarez, municipio de Copala, Guerrero, México.

Datos generales del entrevistado.

Nombre: Edad: Sexo:

Ocupación: Escolaridad:

Tiempo de residencia en la comunidad:

\section{Sistema de producción de ganadería de traspatio.}

¿Qué tipo de animales integran la ganadería de traspatio?

¿Cuantos individuos por especies se localizan en el traspatio?

¿Cuáles son los usos que otorgan a las especies?

¿Cuáles son las partes que utilizan de las especies y como las preparan?

¿Cómo está organizado el trabajo referente al traspatio?

¿Cuál es el papel que desempeña en el traspatio cada uno de los integrantes de la unidad familiar?

¿Cuáles son las actividades que desarrollan cada uno de los miembros de la unidad familiar relacionados con la ganadería familiar?

¿Cuál es el destino de la producción de la ganadería familiar?

¿Cuál es el manejo de la ganadería familiar?

¿Por qué mantienen vigente la cría de animales de traspatio?

¿Cuáles son los problemas por los que ya no crían animales de traspatio? 